Portland State University

PDXScholar

Electrical and Computer Engineering Faculty

Publications and Presentations

Electrical and Computer Engineering

$1-1-2008$

\title{
Statistics of Local Speckle Contrast
}

Donald D. Duncan

Portland State University

Sean J. Kirkpatrick

Ruikang K. Wang

Follow this and additional works at: https://pdxscholar.library.pdx.edu/ece_fac

Part of the Electrical and Computer Engineering Commons

Let us know how access to this document benefits you.

\section{Citation Details}

D. D. Duncan, S. J. Kirkpatrick, and R. K. Wang, "Statistics of local speckle contrast," Journal of the Optical Society of America, A, V. 25, pp. 9-15 (2008).

This Article is brought to you for free and open access. It has been accepted for inclusion in Electrical and Computer Engineering Faculty Publications and Presentations by an authorized administrator of PDXScholar. Please contact us if we can make this document more accessible: pdxscholar@pdx.edu. 


\title{
Statistics of local speckle contrast
}

\author{
Donald D. Duncan,* Sean J. Kirkpatrick, and Ruikang K. Wang \\ Department of Biomedical Engineering, Oregon Health and Science University, 3303 SW Bond Avenue, \\ Portland, Oregon 97239, USA \\ *Corresponding author: donald.duncan@bme.ogi.edu
}

Received August 8, 2007; revised October 16, 2007; accepted October 19, 2007; posted October 19, 2007 (Doc. ID 86233); published December 3, 2007

\begin{abstract}
In describing the first-order properties of laser speckle under polarized illumination conditions, it is almost an article of faith that the contrast is unity. In many processing schemes, however, the contrast defined as the quotient of the standard deviation and the mean is calculated over a localized spatial region. In such cases, this local contrast displays a distribution of values that can depart substantially from unity. Properties of this distribution depend on details of the data acquisition and on the size of the local neighborhood over which the contrast is calculated. We demonstrate that this local contrast can be characterized in terms of a log-normal distribution. Further, we show that the two defining parameters of this model can in turn be expressed in terms of the minimum speckle size and the extent of the local neighborhood. Performance of the model is illustrated with some typical optical coherence tomography data. (C) 2007 Optical Society of America OCIS codes: $030.6140,110.4500,110.6150,120.3890,120.4290,120.6150$.
\end{abstract}

\section{INTRODUCTION}

For a sum of complex field amplitudes with uniformly distributed phases, Goodman [1,2] and others have shown that the intensity, $I$, displays a negative exponential probability density function. As such, the contrast commonly defined as

$$
c=\frac{\sigma_{I}}{\mu_{I}},
$$

is identically equal to unity. In practical applications, e.g., laser speckle imaging (LSI) [3-6], the contrast is calculated [in the manner of Eq. (1)] over a small neighborhood of $p \times p$ pixels. We call this the local speckle contrast. Specifically in LSI, any motion of the speckle pattern during the image acquisition results in a decrease in contrast. Subsequent image segmentation between moving versus stationary, or between regions moving at different velocities, is performed on the basis of differential contrast. Typically, the statistical variation of the local contrast is ignored, and the choice of the size of the local neighborhood is made in an ad hoc fashion [7]. Herein we demonstrate that this local speckle contrast can display values substantially exceeding unity. Further, the local contrast can also exhibit very low values. This should come as no surprise since the theoretical value is based on an analytic distribution that is approached only in the limit of a very large number of samples. In this study we quantify these statistical variations using a series of synthetic speckle patterns.

\section{THEORY}

A spatially band limited speckle pattern can be synthesized easily by the following algorithm (see Fig. 1): fill a circular region of diameter $D$ of a square matrix of dimension $L \times L$ with complex numbers of unity amplitude and with phases uniformly distributed over $(-\pi, \pi)$. Upon Fourier transforming of the $L \times L$ array, and multiplying point-by-point by the complex conjugate, one arrives at a synthetic speckle pattern with exponential probability distribution. The position of the circular region within the larger array is irrelevant as the Fourier shift theorem attests. The ratio of $L$ to $D$ sets the minimum size of the speckles. For example if $D=L$, the Nyquist criterion is met and the smallest speckle is two pixels wide. A speckle pattern generated by this procedure for $L / D=2$ is shown in Fig. 2. For this array of dimension $256 \times 256$, the calculated contrast is 1.0013 . The estimated probability density function (PDF) along with the exponential distribution having the same mean is shown in Fig. 3.

The reason for interest in simulating a band limited speckle pattern is that, in a variety of subsequent processing approaches, one desires that the implicit sampling by the pixels meets the Nyquist criterion, or in a measurement that the minimum speckle size equals the dimen-

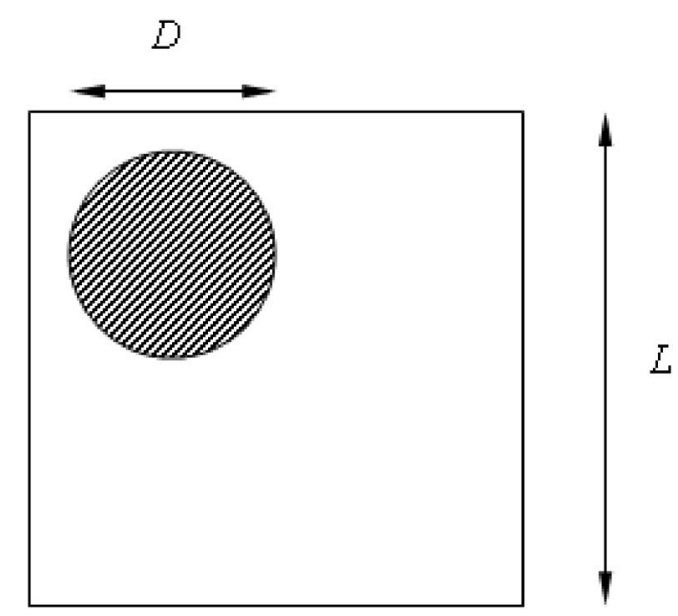

Fig. 1. Illustration of a synthetic speckle algorithm. 


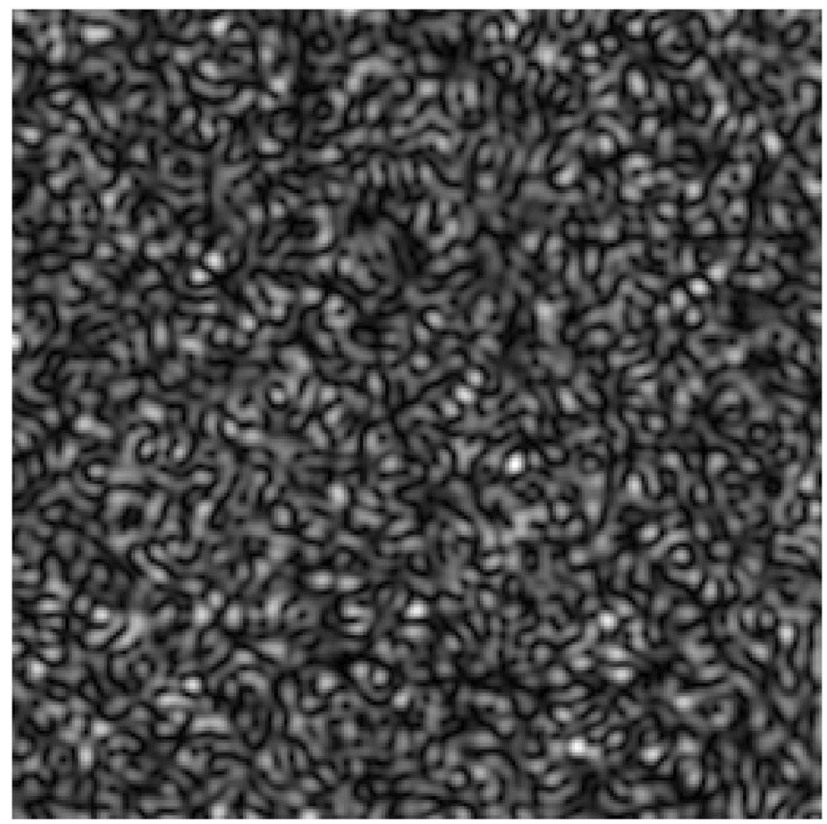

Fig. 2. Example of a synthetic speckle pattern.

sion of the detector [8]. In an experimental situation, various geometrical factors can be manipulated to meet this requirement [1].

Now suppose that we calculate the local speckle contrast over a small local neighborhood, say of $7 \times 7$ pixels. The resulting local contrast image is shown in Fig. 4. One notes that the values span the interval $(0.234,1.98)$. The corresponding contrast probability distribution is shown in Fig. 5. Also shown in this figure is the log-normal distribution defined in terms of the first two moments of the contrast.

$$
\begin{gathered}
f_{C}(c)=\frac{1}{\sqrt{2 \pi} c \ln \sigma_{g}} \exp \left\{-\frac{\ln ^{2}\left(c / c_{m}\right)}{2 \ln ^{2} \sigma_{g}}\right\} ; \\
E\left\{C^{v}\right\}=c_{m}^{v} \exp \left\{\frac{v^{2}}{2} \ln ^{2} \sigma_{g}\right\},
\end{gathered}
$$

where $c_{m}$ is the median (not the mean or the mode) of the distribution and $\sigma_{g}$ is the dimensionless width parameter (not the standard deviation). Note that the normalized variation about the mean contrast (the contrast of the contrast) depends only on the width parameter,

$$
\frac{\sigma_{C}}{\mu_{C}}=\left[\exp \left(\ln ^{2} \sigma_{g}\right)-1\right]^{1 / 2} .
$$

Explicitly, our calculation of local contrast is the quotient of the sample statistics for the mean and standard deviation:

$$
\begin{gathered}
C=\frac{S}{M}, \\
M=\frac{1}{N_{s}} \sum_{i=1}^{N_{s}} I_{i},
\end{gathered}
$$

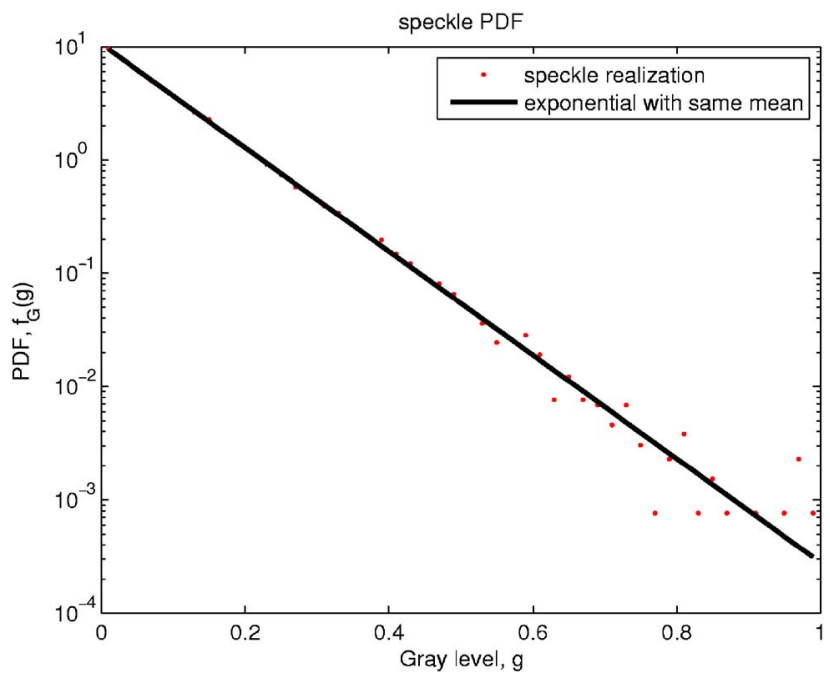

Fig. 3. (Color online) PDF of example speckle pattern and exponential distribution with same mean.

$$
S^{2}=\frac{1}{N_{s}-1} \sum_{i=1}^{N_{s}}\left(I_{i}-M\right)^{2} .
$$

It is easily shown that $M$ and $S$ are unbiased estimators [9], and in the limit of a large number of samples, one would expect convergence to the quotient of the distribution mean and standard deviation,

$$
\frac{S}{M_{N_{s} \rightarrow \infty}} \rightarrow \frac{\sigma}{\mu},
$$

which for an exponential distribution is unity. The calculations of local contrast as described in Eq. (4) are performed easily using the following vectorized MATLAB commands:

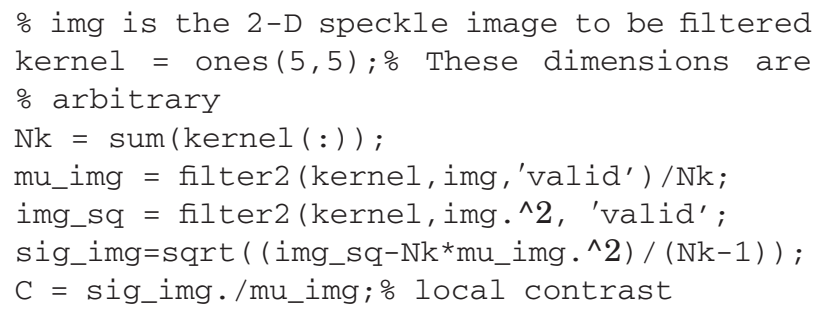

Note that the above is, in effect, a convolutional filtering operation with a nonlinear kernel.

In the preceding equations, and in the subsequent results, we denote random variables with upper case symbols and their realizations with the corresponding lower case symbols. As an example, we use $C$ to denote the contrast random variable and $c$ to denote a particular value (i.e., a number) that the contrast takes on.

\section{RESULTS}

Ordinarily in establishing the PDF of a sample statistic, one would assume statistically independent (SI) draws from the underlying random variable, in this case the intensity, $I_{i}$. In our case, however, these draws are not independent as the low-pass speckle pattern ensures that adjacent pixels are not SI. In fact, it is easily demonstrated 


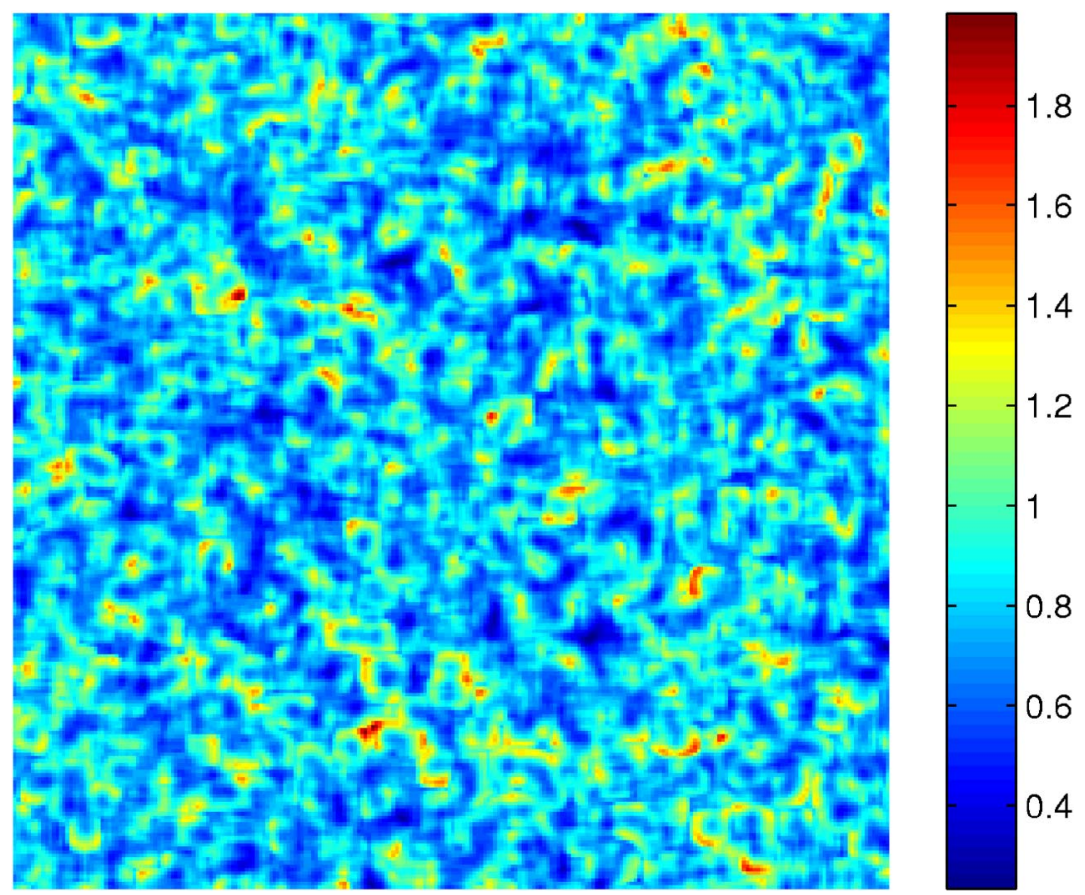

Fig. 4. (Color online) Speckle contrast computed over a $7 \times 7$ pixel region.

that the width of the autocorrelation function of a speckle pattern is on the order of the size of the smallest speckle. Moreover, our situation guarantees that there will be a speckle size dependence on the statistics of the local contrast.

Rather than attempting a theoretical solution to this problem, we take an empirical approach. Specifically, for a series of speckle pattern realizations, we inspect the statistics of the local contrast as calculated over various neighborhood sizes. From a practical standpoint, the size of these local neighborhoods runs from a minimum of nine (for a $3 \times 3$ neighborhood) on up. As such, one could reasonably invoke the central limit theorem, and in the case of a non-negative statistic, the log-normal distribution.

We show in the following two figures the results for SI draws from an exponential distribution. These are the re-

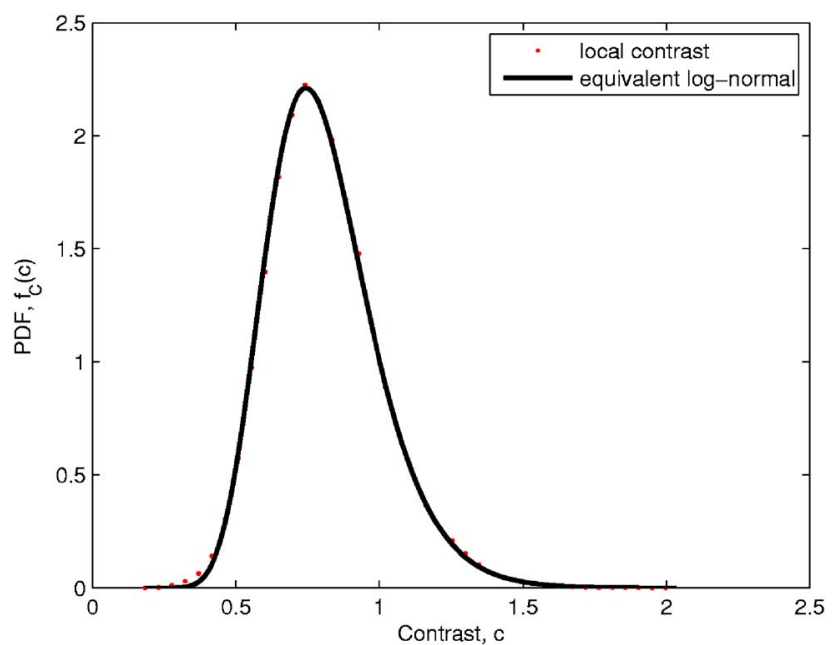

Fig. 5. (Color online) PDF of local contrast and log-normal distribution having same mean and standard deviation (not a fit). sults expected in the limit of no speckle size dependence. Figure 6 shows the median contrast value as a function of the number of samples in a square neighborhood, and Fig. 7 shows the corresponding width parameter. Also shown in these figures are the least squares power law fits.

We note in passing that the normalized variation about the mean contrast for the power law fit in Fig. 7, in the limit of a large number of samples, is [from Eq. (3)]

$$
\frac{\sigma_{C}}{\mu_{C}} \approx 0.86 N_{s}^{-0.46},
$$

which is on the order of $1 / \sqrt{N_{s}}$ as one would expect for independent samples.

Repeating this process for speckle realizations at one, two, and three times the Nyquist sampling rate gives the

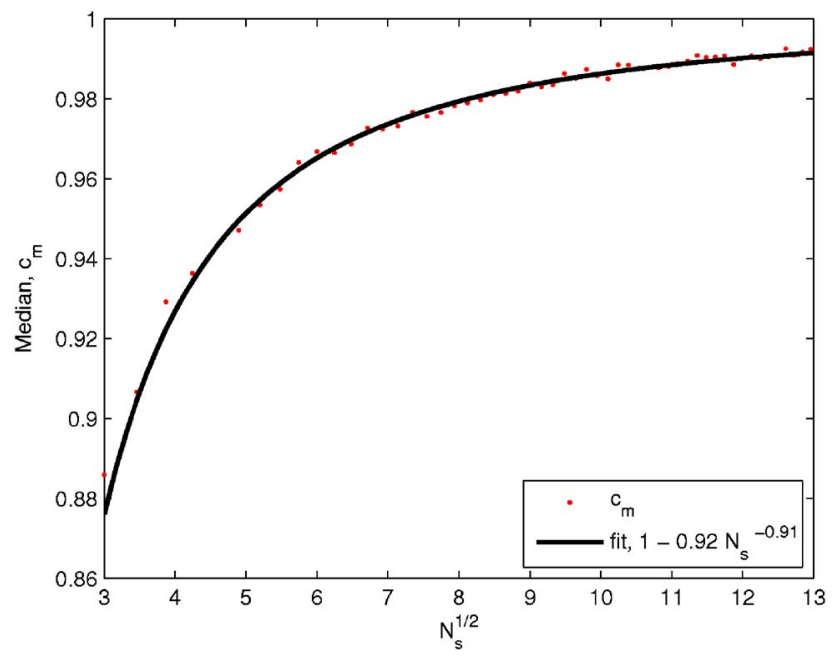

Fig. 6. (Color online) Median parameter of contrast distribution for SI draws from an exponential distribution. 


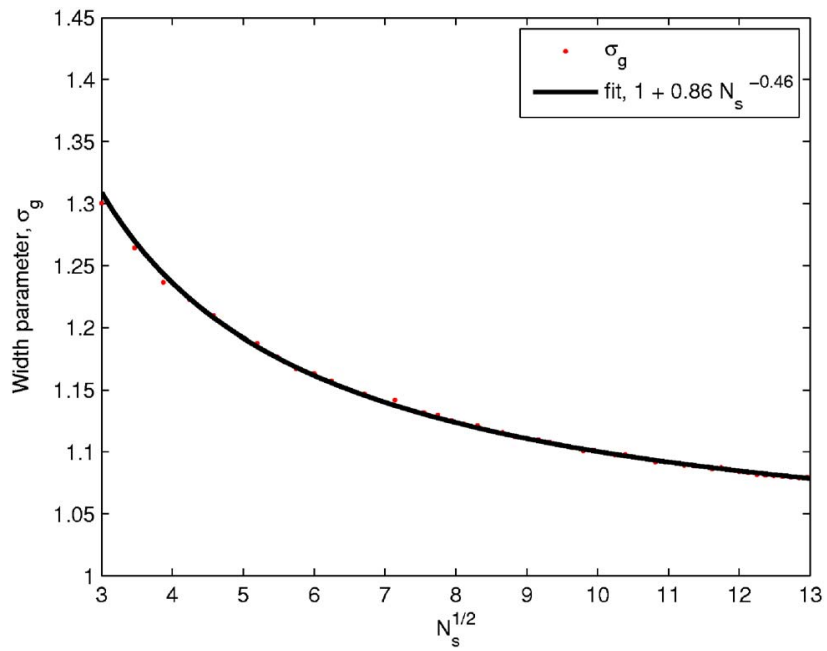

Fig. 7. (Color online) Width parameter of contrast distribution for independent draws from an exponential distribution.

results shown in Fig. 8. As suggested by the results for SI draws from an exponential distribution, the model for each of the fit curves in Fig. 8 is

$$
c_{m}=1-\alpha N_{s}^{\beta} .
$$

Through a bit of trial and error we find that a model that incorporates neighborhood and speckle sizes is of the form

$$
c_{m}=1-\alpha\left(p / N_{s}\right)^{1 / \sqrt{p}},
$$

where $p$ is the number of pixels in the smallest speckle. A Monte Carlo study based on 50 speckle pattern realizations and inspecting a variety of neighborhood sizes yields the two-parameter model,

$$
c_{m}=1-0.734\left(p / N_{s}\right)^{1 / \sqrt{p}} .
$$

Repeating the above process for the width parameter gives the results shown in Fig. 9. Again, as suggested by the results for SI draws from an exponential distribution, the model incorporated in each of these fits is

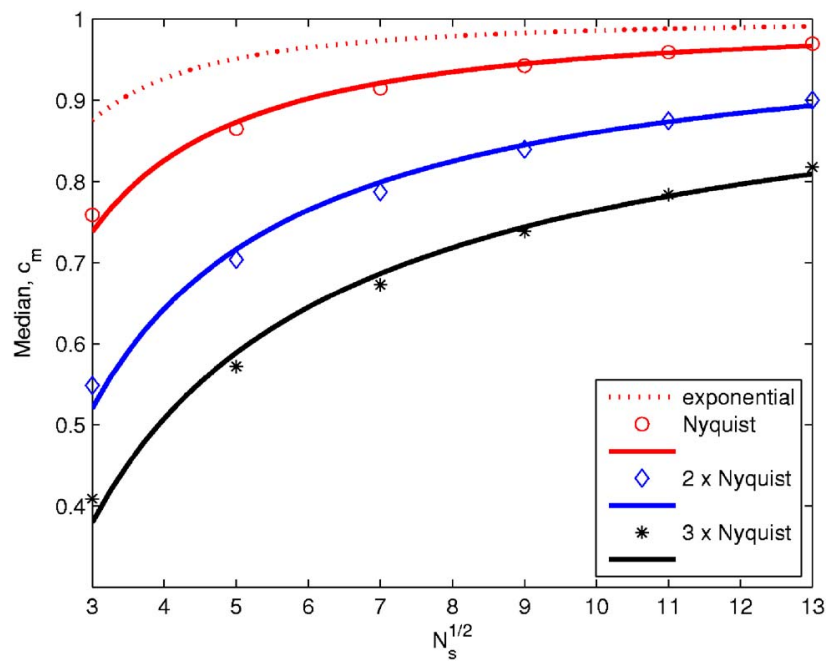

Fig. 8. (Color online) Median parameter of contrast distribution as a function of local neighborhood and speckle size. Dashed curve denoted "exponential" is the result from Fig. 6, shown here for comparison.

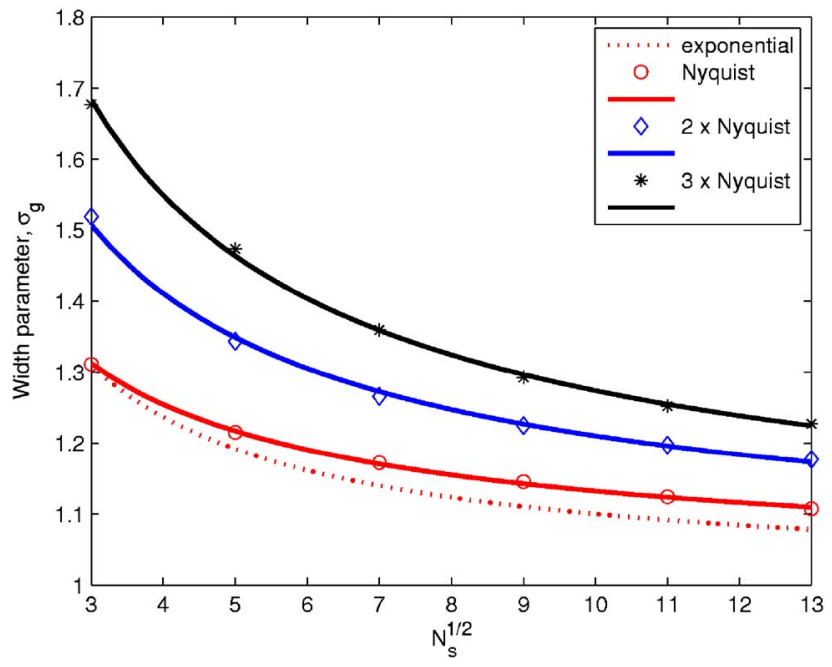

Fig. 9. (Color online) Width parameter for contrast distribution as a function of local neighborhood and speckle size. Dashed curve denoted "exponential" is the result from Fig. 7, shown here for comparison.

$$
\sigma_{g}=1+\alpha N_{s}^{\beta} .
$$

As in the results for the median, the exponent, $\beta$, displays no appreciable variation with the number of pixels, $p$, in the smallest speckle, while the constant parameter, $\alpha$, has a weak dependence on $p$. Monte Carlo simulations suggest a good two-parameter model to be

$$
\sigma_{g}=1+0.454 p^{0.672} N_{s}^{-0.373}
$$

In many coherent imaging modalities, the intensity variations display a Rayleigh distribution instead of an exponential. Such is the case for laser speckle with unpolarized illumination [1]. In this case the theoretical contrast is $1 / \sqrt{2}$ as one would expect for the variance reduction by the number of independent samples in Eq. (2). The previous approach is easily adapted to account for such a situation. Resulting global models for the median and width parameters are the following:

$$
\begin{gathered}
c_{m}=\operatorname{asymptote}\left[1-0.769\left(p / N_{s}\right)^{1.09 / \sqrt{p}}\right], \\
\sigma_{g}=1+0.457 p^{0.697} N_{s}^{-0.382},
\end{gathered}
$$

where asymptote $=1$ and $1 / \sqrt{2}$, respectively, for exponential and gamma intensity distributions (e.g., polarized and unpolarized speckle). The global model for the width parameter is within $\pm 2 \%$ for all cases inspected, and the model for the median parameter is within $+3 \%$ and $-13 \%$. This $13 \%$ model underestimate for the median parameter is observed for the smallest local regions $(3 \times 3)$, but for larger local regions, the errors are within $\pm 3 \%$. Note that the numerical values in Eq. (13) differ slightly from the corresponding values in Eqs. (9) and (11) because of the global fit for both polarized and unpolarized speckle patterns.

Up to this point, there has been no consideration of any additive noise that may accompany the imagery. This is easily accounted for in a practical application with the 


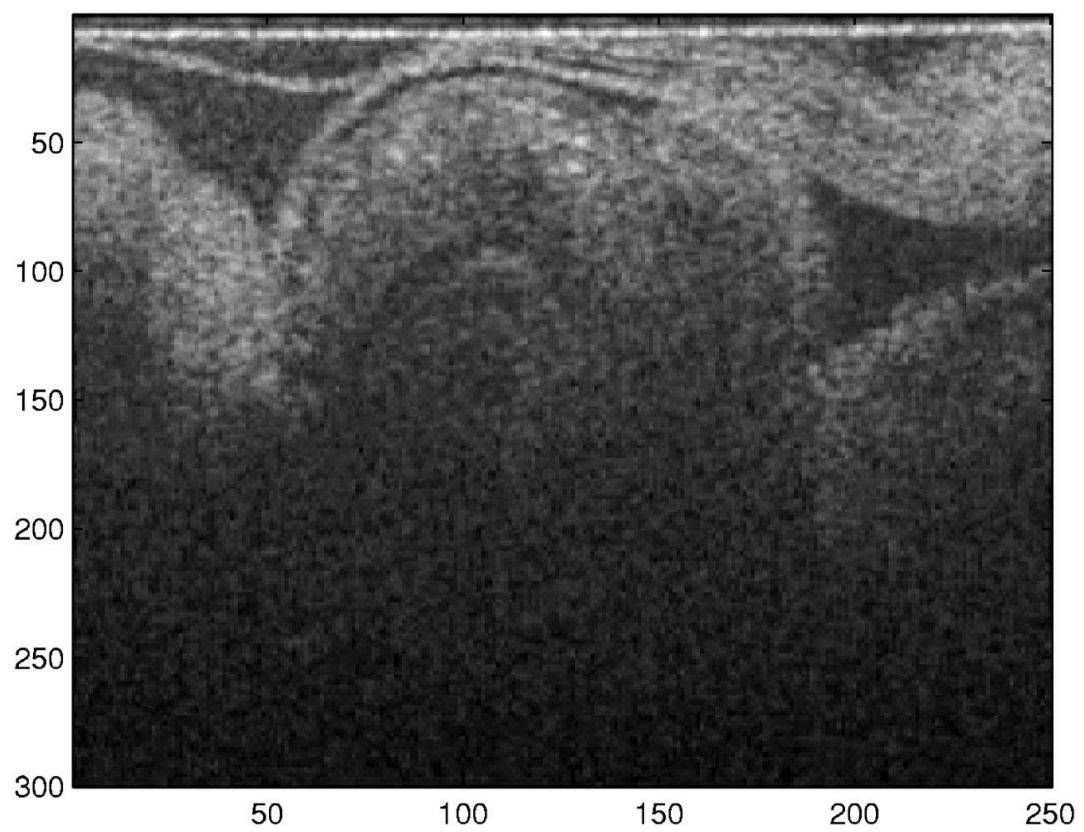

Fig. 10. Single frame of OCT movie of a chick embryo.

addition of a multiplicative factor on the median parameter of

$$
F=\frac{C_{\text {empirical }}}{C_{\text {theoretical }}}
$$

where the denominator is the theoretical global contrast, 1 , or $1 / \sqrt{2}$, for polarized or unpolarized speckle, respectively, and the numerator is the calculated global contrast of the image.

Equations (12) and (13) are the principal results of this study. They give the relationship between the parameters of the local contrast PDF as a function of the size of the neighborhood over which the contrast is estimated and the size of the speckles with respect to the pixels. With these scaling laws, it is possible to optimize an image segmentation that is based on differential contrast. Of course part of this optimization process involves consideration of the trade-off between resolution and neighborhood size. These results provide a logical means of specifying the speckle size for the data acquisition, a local neighborhood size for speckle contrast estimation, and the thresholds to be used in the image segmentation step. Next we illustrate these concepts with a particular example.

As an example of some typical speckle imagery, we present in Fig. 10 a single frame of an optical coherence tomography (OCT) movie of an embryonic chick heart. The gray level PDF is a Rayleigh distribution as one

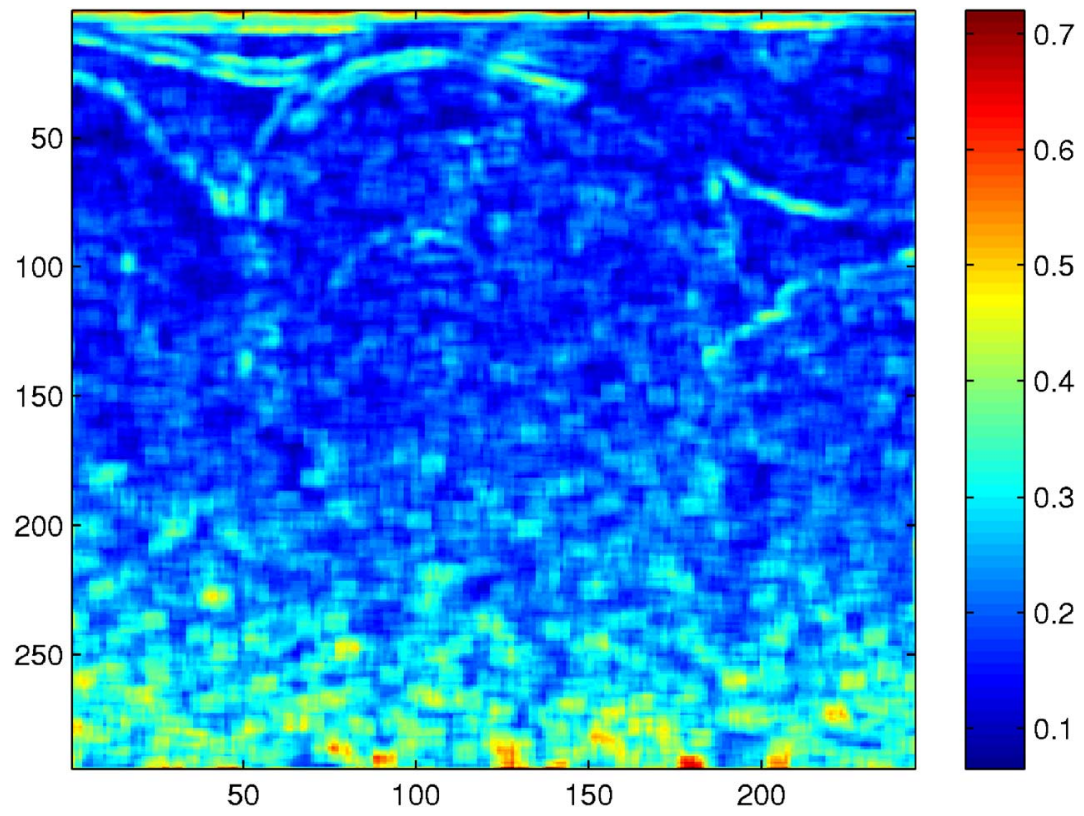

Fig. 11. (Color online) Local contrast computed over a $7 \times 7$ neighborhood. 
would expect for an unpolarized speckle. The global contrast of the entire image is found to be 0.627 . This value reflects not only the structure due to speckle, but also the structure due to the differential scatter within the embryo. A more realistic value for the global contrast due to speckle can be calculated by inspection of the lower portion of the image displaying no image structure; the resulting value of the multiplicative constant [Eq. (13)] is $F=0.517$. Shown in Fig. 11 is the local contrast computed over a $7 \times 7$ region. The $\mathrm{PDF}$ of the speckle was found to have a median value of 0.206 and a width parameter value of 1.42. Inspection of the spatial power spectral density of this image shows that the smallest speckle is approximately 8.5 pixels. Equations (12) predict median and width parameters of 0.220 and 1.46 , respectively, which are in good agreement with the observed parameters.

\section{DISCUSSION AND CONCLUSIONS}

Through the use of a Monte Carlo simulation we have demonstrated that the PDF of local contrast displays a log-normal behavior. This analytic form depends on median and width parameters, which we parameterized on the minimum speckle size, and the number of elements in the local neighborhood over which the contrast is calculated. Next, we discuss the conceptual application of this model to segmentation of an image.

Suppose we have a speckle image as illustrated in Fig. 12. By "speckle image" we mean one acquired by means of some coherent imaging modality. This may be an image that has been acquired with an integration time that is long compared to the motion of the blood within the vessels. As a result, the vessel displays a lower speckle contrast than that of the background. Further suppose that we wish to segment this image into two categories, vessel and background. It makes sense to base this segmentation on the local speckle contrast. The respective speckle contrast distributions may be as illustrated in Fig. 13. From this illustration, it seems obvious what the segmentation threshold should be. However, the subject often occupies a small portion of the image while the majority is background. In such a case the net speckle contrast distribution, for a subject occupying $20 \%$ of the image, might look like that shown in Fig. 14. In this case it is not at all obvious what to choose for the threshold. A parametric model of the distribution of the background speckle con-

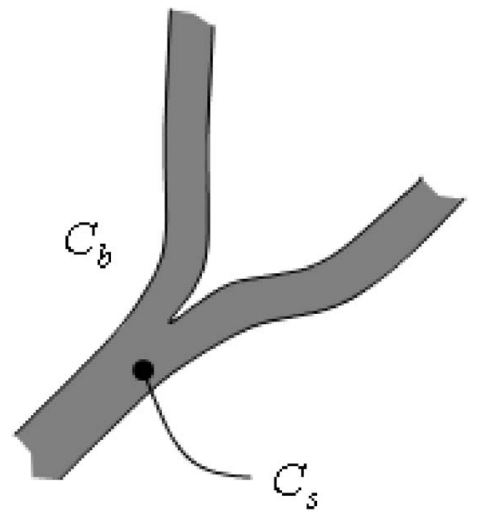

Fig. 12. Image to be segmented.

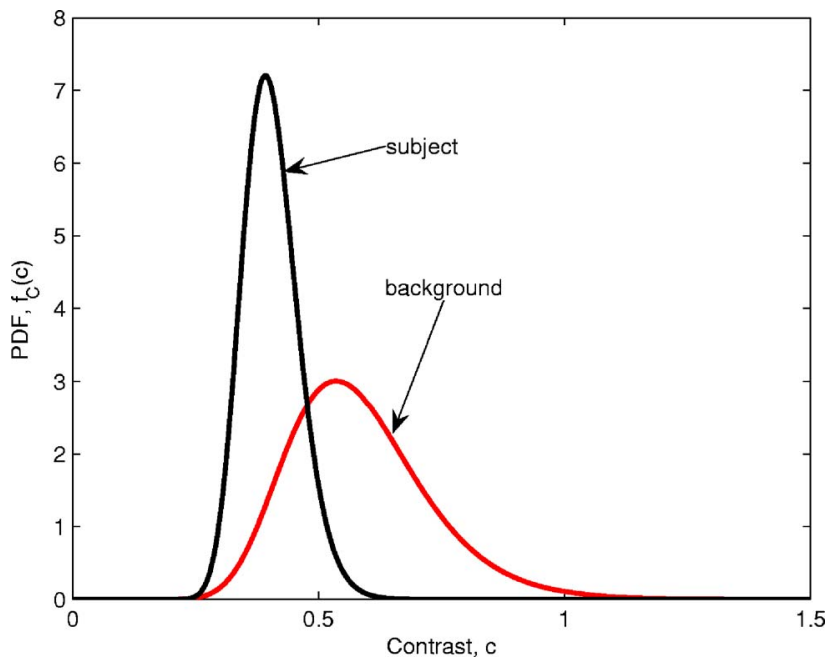

Fig. 13. (Color online) Background and subject contrast PDFs.

trast, however, allows an easy choice of such a threshold. Moreover, this model for the median and width parameters allows one to choose the parameters of the data acquisition (minimum speckle size), and processing (local neighborhood size) to precisely control the properties of the background contrast PDF.

We view this image segmentation step to be the precursor to many problems in which relative motion is to be estimated, for example in LSI [also known as laser speckle contrast analysis (LSCA), or laser speckle spatial contrast analysis (LSSCA)], or its many variants such as laser speckle temporal contrast analysis (LSTCA) [10], or speckle-visibility spectroscopy [8]. Note that Bandyopadhyay et al. [8] correct a long-standing error in the expression for the temporally integrated contrast that seems to date to Fercher and Briers [11] and point out that the assumption of a Lorentzian profile is strictly valid only for Brownian motion.

Once this segmentation step is accomplished, a more detailed analysis can be performed of the statistical variation that takes into consideration the temporal [12] as well as the spatial statistics of the speckle pattern. In this subsequent analysis step, a statistical description of con-

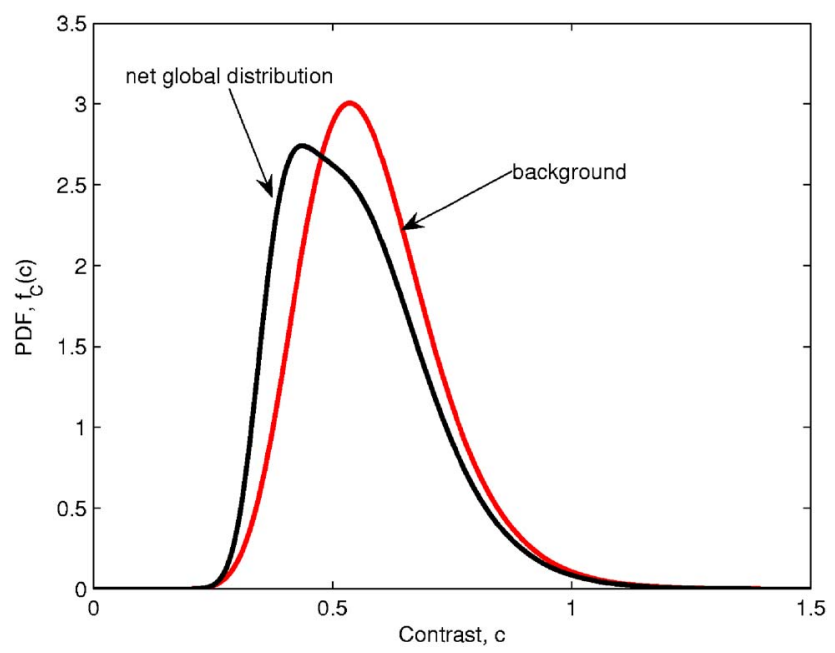

Fig. 14. (Color online) Global contrast distribution compared to that of background. 
trast that takes into consideration both the temporal and spatial variations can be useful. In such a spatiotemporal case, the local speckle contrast is easily calculated with a simple generalization of the previously noted vectorized MATLAB commands:

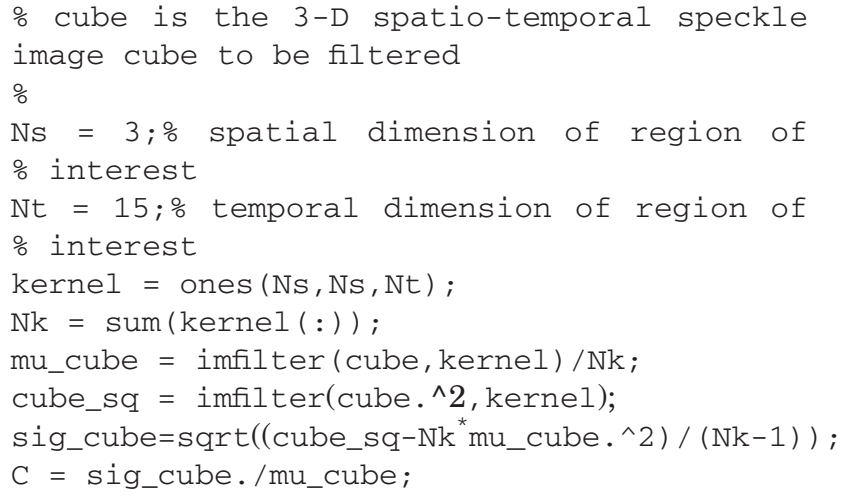

Note that the models discussed herein describe the spatial contrast of a single speckle image while the complementary work described in Kirkpatrick et al. [12] deals strictly with temporal contrast. Ongoing efforts are directed at development of a compressive statistical model of contrast that accounts for both the temporal and spatial variation.

\section{ACKNOWLEDGMENT}

This work was sponsored in part by National Institutes of Health grant CA103824.

\section{REFERENCES}

1. J. W. Goodman, "Statistical properties of laser speckle patterns," in Laser Speckle and Related Phenomena, J. C. Dainty, ed. (Springer-Verlag, 1975), 9-75.

2. J. W. Goodman, Statistical Optics (Wiley \& Sons, 1985).

3. J. D. Briers, "Time-varying laser speckle for measuring motion and flow," Proc. SPIE 4242, 25-39 (2001).

4. T. Durduran, M. G. Burnett, G. Yu, C. Zhou, D. Furuya, A. G. Yodh, J. A. Detre, and J. H. Greenburg, "Spatiotemporal quantification of cerebral blood flow during functional activation in rat somatosensory cortex using laser-speckle flowmetry," J. Cereb. Blood Flow Metab. 24, 518-525 (2004).

5. H. Isono, S. Kishi, Y. Kimura, N. Hagiwara, N. Konishi, and H. Fuji, "Observation of choroidal circulation using index of erythrocytic velocity," Arch. Ophthalmol. (Chicago) 121, 225-231 (2003).

6. P. Zakharov, A. Völker, A. Buck, B. Weber, and F. Scheffold, "Quantitative modeling of laser speckle imaging," Opt. Lett. 31, 3465-3467 (2006).

7. H. Rabal, N. Cap, M. Trivi, R. Arizaga, A. Federico, G. E. Galizzi, and G. H. Kaufmann, "Speckle activity images based on the spatial variance of the phase," Appl. Opt. 45, 8733-8738 (2006).

8. R. Bandyopadhyay, A. S. Gittings, S. S. Suh, P. K. Dixon, and D. J. Durian, "Speckle-visibility spectroscopy: a tool to study time-varying dynamics," Rev. Sci. Instrum. 76, 093110-093110 (2005).

9. A. Papoulis and S. U. Pillai, Probability, Random Variables, and Stochastic Processes, 4th edition (McGraw-Hill, 2001).

10. P. Li, S. Ni, L. Zhang, S. Zeng, and Q. Luo, "Imaging cerebral blood flow through the intact rat skull with temporal laser speckle imaging," Opt. Lett. 31, 1824-1826 (2006).

11. A. F. Fercher and J. D. Briers, "Flow visualization by means of single-exposure speckle photography," Opt. Commun. 37, 326-330 (1981).

12. S. J. Kirkpatrick, D. D. Duncan, R. K. Wang, and M. T. Hinds, "Quantitative temporal speckle contrast imaging for tissue mechanics," J. Opt. Soc. Am. A 24, 3728-3734 (2007). 